\title{
ON THE FRACTIONAL PARTS OF LACUNARY SEQUENCES
}

\author{
ARTŪRAS DUBICKAS
}

\begin{abstract}
In this paper, we prove that if $t_{0}, t_{1}, t_{2}, \ldots$ is a lacunary sequence, namely, $t_{n+1} / t_{n} \geq 1+r^{-1}$ for each $n \geq 0$, where $r$ is a fixed positive number, then there are two positive constants $c(r)=$ $\max \left(1-r, 2(3 r+6)^{-2}\right)$ and $\xi=\xi\left(t_{0}, t_{1}, \ldots\right)$ such that the fractional parts $\left\{\xi t_{n}\right\}, n=0,1,2, \ldots$ all belong to a subinterval of $[0,1)$ of length $1-c(r)$. Some applications of this theorem to the chromatic numbers of certain graphs and to some fast growing sequences are discussed. We prove, for instance, that the number $\sqrt{10}$ can be written as a quotient of two positive numbers whose decimal expansions contain the digits $0,1,2,3$ and 4 only.
\end{abstract}

\section{Introduction}

Sequences of real numbers with 'large gaps' between their consecutive elements are usually called lacunary. In this paper, the sequence of positive real numbers $t_{0}<t_{1}<t_{2}<\cdots$ is called lacunary if there is a real number $a>1$ such that $t_{n+1} / t_{n} \geq a$ for every integer $n \geq 0$. We begin with the following theorem:

THEOREM 1. Let $v$ be a fixed real number, and let $r$ be a fixed positive number. If $t_{0}<t_{1}<t_{2}<\cdots$ is a sequence of positive real numbers satisfying $t_{n+1} \geq\left(1+r^{-1}\right) t_{n}$ for $n=0,1,2, \ldots$ then there is a positive number $\xi$ such that

$$
\left\{\xi t_{n}+v\right\} \leq \min \left(r, 1-2(3 r+6)^{-2}\right)
$$

for each integer $n \geq 0$.

Throughout, for a real number $x$, we denote by $[x],\{x\}$ and $\|x\|$ the integer part of $x$, the fractional part of $x$ and the distance from $x$ to the nearest integer, respectively. In particular, $x=[x]+\{x\}$ and $\|x\|=\min (\{x\}, 1-\{x\})=$ $1 / 2-|1 / 2-\{x\}|$.

The study of fractional and integer parts of lacunary sequences is an old and interesting subject. The first metrical results for the sequences $\left\{\xi a^{n}\right\}$, $n=0,1,2, \ldots$, where $a>1$ were obtained by Weyl [25] and Koksma [17].

Received July 20, 2005. 
Erdôs [14] raised an interesting question in this direction which was answered independently by Pollington [20] and de Mathan [9]. It was also noticed by Erdôs that there is a direct connection between the distribution of fractional parts and the chromatic number of the Cayley graph. This problem was studied by Katznelson [16] and by Ruzsa, Tuza, Voigt [22].

Let $T=\left\{t_{0}=1, t_{1}, t_{2}, \ldots\right\}$ be a lacunary sequence satisfying $t_{n+1} / t_{n} \geq$ $1+r^{-1}$, and let $G$ be an infinite graph, whose vertices are real numbers. The vertices $x$ and $y$ are connected by an edge if and only if $|x-y| \in T$. The chromatic number of $G, \chi(G)$, is the smallest number of colors needed to color $\mathrm{R}$ such that vertices connected by an edge have different colors. In [22] it was proved that $\chi(G) \geq r$ and an upper bound for $\chi(G)$ in terms of $r$ was obtained. Recently, Akhunzhanov and Moshchevitin [3] improved the upper bound to $\chi(G) \leq 2^{7} r^{2}$ for $r \geq 3$. As shown in [22], the estimate $\chi(G) \leq q$ follows directly from the existence of $\xi$ satisfying $\left\|\xi t_{n}\right\| \geq q^{-1}$ for $n=0,1,2, \ldots$. By Theorem 1, there is a positive number $\xi$ such that $\left\|\xi t_{n}\right\| \geq 1 / 9(r+2)^{2}$; this further improves the upper bound to

$$
\chi(G) \leq 9(r+2)^{2}
$$

for each $r \geq 1$.

Another application of the theorem concerns the sequence $t_{n}=n^{\lambda} a^{n}, n=$ $0,1,2, \ldots$, where $a>1$ is a real number. Setting $r:=1 /(a-1)$ and $v:=$ $1-s-\max \left(1-r, 2(3 r+6)^{-2}\right)$ we obtain the following corollary:

Corollary 2. If $a>1$ and $\lambda \geq 0$ are two real numbers, $I=(s, s+$ $\left.\max \left(1-r, 2(3 r+6)^{-2}\right)\right)$ is a subinterval of $[0,1)$, where $r=1 /(a-1)$, then there is a positive number $\xi$ such that the fractional parts $\left\{\xi n^{\lambda} a^{n}\right\}, n=$ $0,1,2, \ldots$, all lie outside $I$.

For a rational number $a>1$, the fractional parts $\left\{\xi a^{n}\right\}, n \geq 0$, were studied in [15], [19], [21], [24] and, more recently, in [5]-[8], [10]-[13]. The case of arbitrary $a>1$ was considered by Tijdeman [23] who showed that there is a positive $\xi$ for which the inequality $\left\{\xi a^{n}\right\} \leq 1 /(a-1), n=0,1,2, \ldots$, holds. (Note that $1-r=(a-2) /(a-1)$, so this result is a part of Corollary 2.) Likewise, the next statement is a corollary of the theorem.

COROLlary 3. If $a>1$ is a real number and $r=1 /(a-1)$, then there is a positive number $\xi$ such that $\left\|\xi a^{n}\right\| \geq \max \left((1-r) / 2,(3 r+6)^{-2}\right)$ for every $n=0,1,2, \ldots$.

Some special cases of this corollary with sharper bounds (in particular, for $a=3 / 2$ ) have been treated in [21] and [12]. In [11] the best possible version of such inequality was obtained for rational integers $a$, See also [8] for another 
best possible result for integer $a>1$, where the answer is given in terms of combinatorics on words [18].

The first inequality of Theorem 1, namely, $\left\{\xi t_{n}+v\right\} \leq r$ follows from the next theorem.

THEOREM 4. Let $v$ be a fixed real number, and let $t_{0}<t_{1}<t_{2}<\cdots$ be a sequence of positive real numbers. Then there is a positive number $\xi$ such that $\left\{\xi t_{n}+v\right\} \leq t_{n} \sum_{j=n+1}^{\infty} t_{j}^{-1}$ for each integer $n \geq 0$.

Indeed, in case $t_{n+1} / t_{n} \geq 1+r^{-1}$, where $n=0,1,2, \ldots$, we get

$$
t_{n} \sum_{j=n+1}^{\infty} t_{j}^{-1} \leq \sum_{j=1}^{\infty}\left(1+r^{-1}\right)^{-j}=r,
$$

so Theorem 4 implies the first inequality of Theorem 1.

Note that the only real solution of $r=1-2(3 r+6)^{-2}$ is $r_{0}=0.9748 \ldots$, so $r<1-2(3 r+6)^{-2}$ for $r<r_{0}$ and $r \geq 1-2(3 r+6)^{-2}$ for $r \geq r_{0}>0.97$. As shown above, for $r<r_{0}<1$, the inequality $\left\{\xi t_{n}+v\right\} \leq r$ of Theorem 1 follows from Theorem 4 whose proof will be given in Section 3. In order to complete the proof of Theorem 1 we will prove in Section 4 that, for $r \geq r_{0}>0.97$, there is a $\xi>0$ such that $\left\{\xi t_{n}+v\right\} \leq 1-2(3 r+6)^{-2}$ for $n=0,1,2, \ldots$ (In the proof of this part, we use the same method of nested intervals as that of Akhunzhanov and Moshchevitin [3] which goes back to [9], [16], [20]; see also [4].)

In the next section, we consider some applications and improvements of the corollaries for the number $a=\sqrt{10}$. Finally, in Section 6 we will apply Theorem 4 to $t_{n}=n$ !.

\section{Decimal expansions of numbers related to $\sqrt{\mathbf{1 0}}$}

The expansions of algebraic numbers in integer bases (in particular, decimal expansions) is another old subject of interest which is related to the distribution of fractional parts of powers. See, for instance, [1], [2] for a recent progress on complexity of such expansions.

It is well-known that each real number $\zeta$ can be written in the form $g+$ $0 . g_{1} g_{2} g_{3} \ldots=g+\sum_{j=1}^{\infty} g_{j} / 10^{j}$, where $g=[\zeta] \in \mathbf{Z}$ and $g_{1}, g_{2}, g_{3}, \ldots \in$ $\{0,1, \ldots, 9\}$. (If we ban an infinite string of 9 , then such a decimal expansion of $\zeta$ is unique.) We say that the decimal expansion $g+0 . g_{1} g_{2} g_{3} \ldots \in \mathrm{R}$ has digits in the set $D$ if $\left\{g_{1}, g_{2}, g_{3}, \ldots\right\} \subseteq D$.

Let $u$ be a fixed integer in the set $\{0,1, \ldots, 9\}$ and let $D_{u}$ be the set of five consecutive integers $u, \ldots, u+3, u+4$ modulo 10 . For instance, $D_{0}=$ $\{0,1,2,3,4\}$ and $D_{8}=\{0,1,2,8,9\}$. 
THEOREM 5. Let $u$ be a fixed integer satisfying $0 \leq u \leq 9$. The number $\sqrt{10}$ can be written as a quotient of two positive numbers whose decimal expansions have digits in $D_{u}$.

Note that Corollary 2 implies that there is a positive number $\xi$ such that $\left\{\xi 10^{n / 2}\right\} \leq(\sqrt{10}+1) / 9=0.462475 \ldots$. The next corollary improves this bound to $4 / 9=0.444444 \ldots$.

Corollary 6. Let $u$ be a fixed integer satisfying $0 \leq u \leq 5$. Then there is a positive number $\xi$ such that $u / 9 \leq\left\{\xi 10^{n / 2}\right\} \leq(u+4) / 9$ for each integer $n \geq 0$.

As for the distance the nearest integer, Corollary 3 implies that there is a positive $\xi$ such that $\left\|\xi 10^{n / 2}\right\| \geq(\sqrt{10}-2) / 2(\sqrt{10}-1)=0.268762 \ldots$ We improve this constant to $3 / 11=0.272727 \ldots$.

COROLLARY 7. There is a positive integer $\xi$ such that $\left\|\xi 10^{n / 2}\right\| \geq 3 / 11$ for each integer $n \geq 0$. Moreover, with the same $\xi$ we have $\left\|\xi 10^{n / 2}\right\| \geq 1 / 3$ for each integer $n \geq 0$, unless there exists an integer $h \geq 0$ such that the decimal expansion of the number $(h+3 / 11) \sqrt{10}$ contains no digits $0,1,8,9$.

Apparently, there is no $h \in \mathrm{N}$ for which the decimal expansion of the number $(h+3 / 11) \sqrt{10}$ does not contain the digits $0,1,8,9$. This would follow from a natural (but out of reach) conjecture which claims that every irrational algebraic number is normal in every integer base. If so, then the decimal expansion of an algebraic irrational number (in particular, $(h+3 / 11) \sqrt{10}$ ) has infinitely many digits equal to 0 .

Naturally, Theorem 5 and Corollaries 6 and 7 can be stated for the number $\sqrt{b}$ in base $b$, where $b \geq 5$ is an integer (see Section 5). However, we will conclude with one more statement in base 10 , where the cubic root of 10 is considered instead of the square root.

THEOREM 8. Let $u$ be a fixed number in $\{0,1, \ldots, 9\}$. There is positive number $\xi$ such that the digit $u$ does not appear in the decimal expansions of the numbers $\xi, \xi 10^{1 / 3}$ and $\xi 10^{2 / 3}$.

All results stated in this section will be proved in Section 5 .

\section{Proof of Theorem 4}

Let $k_{0}>v$ be an integer. Set $T_{n}:=t_{n} \sum_{j=n+1}^{\infty} t_{j}^{-1}$ for $n=0,1,2, \ldots$ and $I_{0}=\left[\left(k_{0}-v\right) / t_{0},\left(k_{0}-v+T_{0}\right) / t_{0}\right]$. It is clear that $\left\{\zeta t_{0}+v\right\} \leq T_{0}$ for each $\zeta \in I_{0}$. Suppose we have $n$ closed intervals $I_{n-1} \subseteq I_{n-2} \subseteq \cdots \subseteq I_{0}$, where $I_{j}=\left[\left(k_{j}-v\right) / t_{j},\left(k_{j}-v+T_{j}\right) / t_{j}\right], k_{j} \in \mathbf{Z}$, such that for each $\zeta \in I_{n-1}$ the inequality $\left\{\zeta t_{j}+\nu\right\} \leq T_{j}$ holds for every $j=0,1, \ldots, n-1$. We claim that 
there is an interval $I_{n}=\left[\left(k_{n}-v\right) / t_{n},\left(k_{n}-v+T_{n}\right) / t_{n}\right] \subseteq I_{n-1}$ with $k_{n} \in \mathrm{Z}$, such that $\left\{\zeta t_{n}+v\right\} \leq T_{n}$ for each $\zeta \in I_{n}$.

Note that $\left\{\zeta t_{n}+v\right\} \leq T_{n}$ if and only if there is an integer $k_{n}$ such that $k_{n} \leq \zeta t_{n}+v \leq k_{n}+T_{n}$, that is, $\zeta \in\left[\left(k_{n}-v\right) / t_{n},\left(k_{n}-v+T_{n}\right) / t_{n}\right]=$ $I_{n}$. Clearly, $I_{n} \subseteq I_{n-1}$ if and only if $\left(k_{n}-v\right) / t_{n} \geq\left(k_{n-1}-v\right) / t_{n-1}$ and $\left(k_{n}-v+T_{n}\right) / t_{n} \leq\left(k_{n-1}-v+T_{n-1}\right) / t_{n-1}$. Setting $s_{n}:=\left(k_{n-1}-v\right) t_{n} / t_{n-1}+v$, we see that these inequalities hold if

$$
s_{n} \leq k_{n} \leq\left(k_{n-1}-v+T_{n-1}\right) t_{n} / t_{n-1}+v-T_{n}=s_{n}+1 .
$$

Here, we used the equality $t_{n} T_{n-1} / t_{n-1}-T_{n}=1$. The interval $\left[s_{n}, s_{n}+1\right]$ is of length 1 , so it indeed contains an integer $k_{n}$, as claimed.

This proves that, for every $\zeta \in I_{n}$, we have $\left\{\zeta t_{j}+v\right\} \leq T_{j}$ for each $j=0,1, \ldots, n$. The number $\xi=\cap_{n=0}^{\infty} I_{n}$ satisfies the required condition: $\left\{\xi t_{n}+v\right\} \leq T_{n}$ for $n=0,1,2, \ldots$. Clearly, $\xi$ is positive, because so is the left endpoint of $I_{0}$.

\section{Proof of Theorem 1}

In the sequel, let

$$
\begin{aligned}
g & :=[(7 / 2)(r+1) \log (r+2)]+1, \\
w & :=(2 / 9)(r+2)^{-2}, \\
\delta & :=\sqrt{w(w+1) r(r+1)}=\frac{\sqrt{2 r(r+1)\left(1+(2 / 9)(r+2)^{-2}\right)}}{3(r+2)} .
\end{aligned}
$$

Note that $w<0.03$ and $\delta<\sqrt{2} / 3<1 / 2$ for $r \geq 0.97$.

We will construct the sequence of closed nested intervals $I_{0}, I_{1}, I_{2}, \ldots$, where $\left|I_{m}\right|=\delta / r t_{g m}(|I|$ stands for the length of $I), I_{m+1} \subset I_{m}$ for each $m \geq 0$, and $\left\{\zeta t_{n}+\nu\right\} \leq 1-w$ for every $\zeta \in I_{m}$ and $n=-1,0,1, \ldots, g m-1$. (For convenience, an additional term $t_{-1}:=t_{0} /\left(1+r^{-1}\right)$ is added to the sequence.) The proof is by induction on $m$.

We begin with $m=0$. Note that $\delta / r<1 / 2 r<0.52<1-w$ for $r \geq 0.97$. Hence we can take any subinterval of length $\delta / r t_{0}<\delta / r t_{-1}$ of the interval $\left[\left(k_{-1}-v\right) / t_{-1},\left(k_{-1}-v+1-w\right) / t_{-1}\right]$ of length $(1-w) / t_{-1}$, where $k_{-1}>v$ is an arbitrary integer.

For the induction step $m \rightarrow m+1$, let us suppose that there exist intervals $I_{m} \subset I_{m-1} \subset \ldots \subset I_{0}$ such that $\left\{\zeta t_{n}+v\right\} \leq 1-w$ for every $\zeta \in I_{m}$ and $n=-1,0,1, \ldots, g m-1$. It remains to show that there is an interval $I_{m+1} \subset I_{m}$ of length $\delta / r t_{g(m+1)}$ such that $\left\{\zeta t_{n}+v\right\} \leq 1-w$ for each $\zeta \in$ $I_{m+1}$ and $n=g m, g m+1, \ldots, g(m+1)-1$. Write $I_{m}=\left[v, v^{\prime}\right]$. For each 
$n \in\{g m, g m+1, \ldots, g(m+1)-1\}$, let $S_{n}$ be the set of points of the form $(k-v-w) / t_{n}$, where $k \in \mathbf{Z}$, in the interval $\left[v-w / t_{n}, v^{\prime}\right]$. There are at most $1+w+t_{n}\left|I_{m}\right|$ such points; at most $1+t_{n}\left|I_{m}\right|$ of them belong to the interval $I_{m}$.

Let $J_{n}$ be the union of all intervals $\left(s, s+w / t_{n}\right)$, where $s \in S_{n}$. For each point $\zeta$ of $I_{m}$ which is outside the union of intervals $\cup_{n=g m}^{g(m+1)-1} J_{n}$ we clearly have $\left\{\zeta t_{n}+v\right\} \leq 1-w$. So it remains to show that $I_{m}-\cup_{n=g m}^{g(m+1)-1} J_{n}$ contains a closed interval of length $\delta / r t_{g(m+1)}$. The sum of lengths of all intervals in $\cup_{n=g m}^{g(m+1)-1} J_{n}$ does not exceed

$$
\begin{aligned}
\sum_{n=g m}^{g(m+1)-1}\left(1+w+t_{n}\left|I_{m}\right|\right) w / t_{n} & =g w\left|I_{m}\right|+w(1+w) \sum_{n=g m}^{g(m+1)-1} t_{n}^{-1} \\
& <g w\left|I_{m}\right|+\frac{w(1+w)}{t_{g m}} \sum_{j=0}^{\infty}\left(1+r^{-1}\right)^{-j} \\
& =g w\left|I_{m}\right|+w(1+w)(r+1) / t_{g m} \\
& =(g w+w(1+w) r(1+r) / \delta)\left|I_{m}\right| \\
& =(g w+\delta)\left|I_{m}\right| .
\end{aligned}
$$

The length of the remaining part in $I_{m}$ (which is a union of closed intervals, possibly degenerate intervals $[v, v]$ of length zero) is greater than

$$
\left|I_{m}\right|-(g w+\delta)\left|I_{m}\right|=(1-g w-\delta)\left|I_{m}\right|=(1-g w-\delta) \delta / r t_{g m} .
$$

The number of intervals in $\cup_{n=g m}^{g(m+1)-1} J_{n}$ that have non-empty intersection with $I_{m}$ can be bounded above by

$$
\begin{aligned}
\sum_{n=g m}^{g(m+1)-1}\left(1+t_{n}\left|I_{m}\right|\right) & =g+\left|I_{m}\right| \sum_{n=g m}^{g(m+1)-1} t_{n} \\
& <g+\left|I_{m}\right| t_{g(m+1)} \sum_{j=1}^{\infty}\left(1+r^{-1}\right)^{-j} \\
& =g+r t_{g(m+1)}\left|I_{m}\right| .
\end{aligned}
$$

Hence the set $I_{m}-\cup_{n=g m}^{g(m+1)-1} J_{n}$ is an interval from which at most $g+$ $r t_{g(m+1)}\left|I_{m}\right|$ open intervals are removed. Thus it consists of at most $1+g+$ $r t_{g(m+1)}\left|I_{m}\right|=1+g+\delta t_{g(m+1)} / t_{g m}$ closed intervals.

We claim that at least one of them is of length $\geq \delta / r t_{g(m+1)}$. Clearly, this is the case if $\left((1-g w-\delta) \delta / r t_{g m}\right) /\left(1+g+\delta t_{g(m+1)} / t_{g m}\right) \geq \delta / r t_{g(m+1)}$, that is, 
$t_{g(m+1)}(1-g w-2 \delta) \geq t_{g m}(1+g)$. Using $t_{g(m+1)} / t_{g m} \geq\left(1+r^{-1}\right)^{g}$ we see that the required inequality holds if

$$
g w+2 \delta+(g+1)\left(1+r^{-1}\right)^{-g}<1 .
$$

Estimating $\left(1+r^{-1}\right)^{g} \geq\left(1+r^{-1}\right)^{(7 / 2)(r+1) \log (r+2)}>e^{(7 / 2) \log (r+2)}=(r+2)^{7 / 2}$ and $g+1 \leq(7 / 2)(r+1) \log (r+2)+2$, we obtain $(g+1)\left(1+r^{-1}\right)^{-g}<$ $((7 / 2)(r+1) \log (r+2)+2)(r+2)^{-7 / 2}$. Next, using $g \leq(7 / 2)(r+1) \log (r+$ $2)+1$ and expressing $w, \delta$ in terms of $r$, we deduce that the required inequality holds if

$$
\begin{aligned}
& \frac{7(r+1) \log (r+2)+2}{9(r+2)^{2}}+\frac{7(r+1) \log (r+2)+4}{2(r+2)^{7 / 2}} \\
&+\frac{2 \sqrt{\left(2+(4 / 9)(r+2)^{-2}\right)\left(r^{2}+r\right)}}{3(r+2)}<1 .
\end{aligned}
$$

By an easy computation we see that this inequality holds for every $r \geq 0.97$; in fact, the maximum of the left hand side in the interval $[0.97, \infty)$ occurs at approximately $r=12.2 \ldots$ and is equal to $0.9907 \ldots$.

\section{Fractional parts of powers of $\sqrt{\mathbf{1 0}}$}

Proof of Theorem 5. Fix $u$ in $\{0,1, \ldots, 9\}$ and set $t_{n}=10^{n / 2}, v=-u / 10$ in Theorem 1. Note that $0.4625>1 /(\sqrt{10}-1)$. It follows from Theorem 1 that there is a positive $\xi$ such that $\left\{\xi 10^{n / 2}-u / 10\right\}<0.4625$ for each integer $n \geq 0$. Hence $\left\{\xi 10^{k}-u / 10\right\}<1 / 2$ and $\left\{(\xi \sqrt{10}) 10^{k}-u / 10\right\}<1 / 2$ for each integer $k \geq 0$. Thus the only digits that can appear in the decimal expansions of $\xi$ and $\xi \sqrt{10}$ are $u$ and $u+1, \ldots, u+4$ modulo 10 , that is, those belonging to $D_{u}$. This proves Theorem 5 .

Proof of Corollary 6. Fix $u$ in $\{0,1,2,3,4,5\}$. By Theorem 5, there is a positive number $\xi$ such that both $\xi$ and $\xi \sqrt{10}$ have the digits $u, u+1, \ldots, u+4$ in their decimal expansions only. Thus the smallest possible value for $\left\{\xi 10^{k}\right\}$ and $\left\{(\xi \sqrt{10}) 10^{k}\right\}, k \geq 0$, is $0 . u u u \ldots=u / 9$ and the largest value is $(u+$ 4) $/ 9$ (The largest value $(u+4) / 9=1$ is not attained for $u=5$, because a decimal expansion cannot end by an infinite string of 9.) It follows that $u / 9 \leq\left\{\xi 10^{n / 2}\right\} \leq(u+4) / 9$ for each integer $n \geq 0$.

By considering the integer base $b \geq 5$, we will combine a part of Theorem 5 and a part of Corollary 6 into the following statement:

THEOREM 9. Let $b \geq 5$ be an integer which is not a square, and let $u \leq$ $b-[b /(\sqrt{b}-1)]-1$ be a fixed non-negative integer. Then the number $\sqrt{b}$ can be written as a quotient of two positive numbers whose expansions in base $b$ 
have digits in $\{u, u+1, \ldots, u+[b /(\sqrt{b}-1)]\}$ only. Furthermore, there is a positive number $\xi$ such that $\left\{\xi b^{n / 2}\right\} \leq[b /(\sqrt{b}-1)] /(b-1)$ for each integer $n \geq 0$.

The last inequality improves the upper bound $\left\{\xi b^{n / 2}\right\} \leq(1+\sqrt{b}) /(b-1)$ which comes from Corollary 2 in case $[b /(\sqrt{b}-1)]<1+\sqrt{b}$. (This inequality can be false; $b=10$ is the smallest integer which for which this inequality holds.) The proofs of both statements of Theorem 9 are exactly the same as those of Theorem 5 and Corollary 6. By Theorem 1, observing that there is a positive number $\xi$ such that $\left\{\xi b^{n / 2}-u /(b-1)\right\} \leq 1 /(\sqrt{b}-1)$ for each integer $n \geq 0$, one can derive easily that the $b$-adic expansions of $\xi$ and $\xi \sqrt{b}$ cannot have digits other than $u, u+1, \ldots, u+[b /(\sqrt{b}-1)]$. The details are left to the reader.

Proof of Corollary 7. By Theorem 1 again, there is a positive number $\xi$ such that $0.2687<\left\{\xi 10^{n / 2}\right\}<0.7313$ for each integer $n \geq 0$. Thus $0.2687<$ $\left\{\xi 10^{k}\right\}<0.7313$ and $0.2687<\left\{(\xi \sqrt{10}) 10^{k}\right\}<0.7313$ for each integer $k \geq 0$. It follows that the numbers $\xi$ and $\xi \sqrt{10}$ have only digits $2,3,4,5,6,7$ in their decimal expansions. Furthermore, each digit 2 in such an expansion should be followed by the digit 7 . Indeed the next digit cannot be smaller than 6 ; but if it is 6 , then the next digit must be either 8 or 9 , a contradiction. So each 2 must be followed by 7. Similarly, upper bounds show that each digit 7 must be followed by 2 .

We thus arrive to the following two alternatives. Either the numbers $\xi$ and $\xi \sqrt{10}$ have only digits $3,4,5,6$ in their decimal expansions or those digits are $2,3,4,5,6,7$ and at least one of the numbers $\xi, \xi \sqrt{10}$ is equal to $g+0 . g_{1} \ldots g_{m} 272727 \ldots=g+\frac{g_{1}}{10}+\cdots+\frac{g_{m}}{10^{m}}+\frac{3}{11 \cdot 10^{m}}=(h+3 / 11) 10^{-m}$,

where $h$ and $m$ are non-negative integers. In the first case, there is a positive integer $\xi$ such that $0.3333 \ldots=1 / 3 \leq\left\{\xi 10^{n / 2}\right\} \leq 2 / 3=0.6666 \ldots$, so that $\left\|\xi 10^{n / 2}\right\| \geq 1 / 3$ for each integer $n \geq 0$.

In the second case, suppose, for instance, that $\xi \sqrt{10}=(h+3 / 11) 10^{-m}$. (The case when $\xi=(h+3 / 11) 10^{-m}$ can be treated similarly.) Then $\xi$ is irrational, so its decimal expansion does not contain the digits 2 and 7 , because otherwise $\xi$ should be of the form $\left(h^{\prime}+3 / 11\right) 10^{-m^{\prime}}$ too. Hence, on replacing $\xi$ by $\xi^{\prime}=\xi 10^{m}$, we obtain that $\left\{\xi^{\prime} 10^{k}\right\} \in[1 / 3,2 / 3]$ for each integer $k \geq 0$ and that $\left\{\left(\xi^{\prime} \sqrt{10}\right) 10^{k}\right\}$ takes only two values, namely, $0.2727 \ldots=3 / 11$ and $0.7272 \ldots=8 / 11$ for each integer $k \geq 0$. Hence

$$
\left\|\xi^{\prime} 10^{n / 2}\right\| \in\{3 / 11\} \cup[1 / 3,1 / 2]
$$

for each integer $n \geq 0$. 
It follows that in both cases there is a positive $\xi$ for which $\left\|\xi 10^{n / 2}\right\| \epsilon$ $\{3 / 11\} \cup[1 / 3,1 / 2], n=0,1,2, \ldots$, which yields the inequality $\left\|\xi 10^{n / 2}\right\| \geq$ $3 / 11$, as claimed. Note that, with this $\xi$, using $\xi \sqrt{10}=(h+3 / 11) 10^{-m}$ we can write $(h+3 / 11) \sqrt{10}=\xi 10^{m+1}$. From the fact that the decimal expansion of $\xi$ contains the digits 2, 3, 4, 5, 6, 7 only, we conclude that there is an integer $h \geq 0$ such that the decimal expansion of $(h+3 / 11) \sqrt{10}$ contains no digits $0,1,8,9$. If such $h$ as claimed in Corollary 7 does not exist, then the second alternative cannot occur and so the stronger inequality $\left\|\xi 10^{n / 2}\right\| \geq 1 / 3$ holds for each integer $n \geq 0$.

Proof of Theorem 8. Fix $u$ in $\{0,1, \ldots, 9\}$ and set $t_{n}=10^{n / 3}, v=$ $-(u+1) / 10$ in Theorem 1 . Note that $0.9>1 /\left(10^{1 / 3}-1\right)$. It follows from Theorem 1 that there is a positive $\xi$ such that $\left\{\xi 10^{n / 3}-(u+1) / 10\right\}<0.9$ for each integer $n \geq 0$. The number $\xi$ satisfies the conditions of the theorem. Indeed, since $\left\{\xi 10^{k}-(u+1) / 10\right\}<0.9,\left\{\left(\xi 10^{1 / 3}\right) 10^{k}-(u+1) / 10\right\}<0.9$ and $\left\{\left(\xi 10^{2 / 3}\right) 10^{k}-(u+1) / 10\right\}<0.9$ for each integer $k \geq 0$, the digit $u$ cannot appear in the decimal expansions of $\xi$, $\xi 10^{1 / 3}$ and $\xi 10^{2 / 3}$.

\section{Fast growing sequences}

Let us apply Theorem 4 to $t_{n}=r_{1} r_{2} \ldots r_{n}$, where $1 \leq r_{0} \leq r_{1} \leq r_{2} \leq \ldots$ is an arbitrary sequence of positive real numbers satisfying $r_{n} \rightarrow \infty$ as $n \rightarrow \infty$. Since

$$
T_{n}=t_{n} \sum_{j=n+1}^{\infty} t_{j}^{-1}=\sum_{j=1}^{\infty}\left(r_{n+1} r_{n+2} \ldots r_{n+j}\right)^{-1}<\sum_{j=1}^{\infty} r_{n+1}^{-j}=1 /\left(r_{n+1}-1\right),
$$

Theorem 4 implies that there is a $\xi>0$ such that

$$
\left\{\xi r_{0} r_{1} \ldots r_{n}+v\right\}<1 /\left(r_{n+1}-1\right)
$$

for $n=0,1,2, \ldots$ In particular, for any $\eta \in[0,1]$, there is a $\xi>0$ such that

$$
\lim _{n \rightarrow \infty}\left\{\xi r_{0} r_{1} \ldots r_{n}\right\}=\eta
$$

Likewise, on applying the above result to $r_{n}=n$, we derive that there is a $\xi>0$ such that $\{\xi n !+\nu\}<1 / n$ for $n=1,2, \ldots$ Next, putting $\nu=1 / 2$, we derive that there is a $\xi>0$ such that

$$
\{\xi n !\} \in(1 / 2,1 / 2+1 / n)
$$

for each $n=2,3, \ldots$. (Plainly, such $\xi$ should be irrational.) In particular, this implies that $\|\xi n !\|>1 / 2-1 / n$ for each $n=2,3, \ldots$. 
On the other hand, we claim that, for any real $\xi$, the inequality

$$
\|\xi n !\|<1 / 2-1 / 2(n+2)
$$

holds for infinitely many $n \in \mathrm{N}$.

Indeed, writing, for each $n \in \mathbf{N}, \xi n !=x_{n}+1 / 2+z_{n}$, where $x_{n} \in \mathbf{Z}$ and $z_{n} \in[-1 / 2,1 / 2)$, we have

$$
(n+1)\left(x_{n}+1 / 2+z_{n}\right)=x_{n+1}+1 / 2+z_{n+1} .
$$

Hence

$$
(n+1) x_{n}+n / 2-x_{n+1}=z_{n+1}-(n+1) z_{n} .
$$

It follows that $\left|z_{n+1}-(n+1) z_{n}\right| \geq 1 / 2$ for each odd $n$. If both $\left|z_{n}\right| \leq 1 / 2(n+2)$ and $\left|z_{n+1}\right| \leq 1 / 2(n+3)$ hold, then

$$
\begin{aligned}
1 / 2 \leq\left|z_{n+1}\right|+(n+1)\left|z_{n}\right| \leq & 1 / 2(n+3)+(n+1) / 2(n+2) \\
& =\left(n^{2}+5 n+5\right) / 2\left(n^{2}+5 n+6\right)<1 / 2,
\end{aligned}
$$

a contradiction. So either $\left|z_{n}\right|>1 / 2(n+2)$ or $\left|z_{n+1}\right|>1 / 2(n+3)$. Consequently, for any $M \in \mathrm{N}$, the inequality $\|\xi n !\|=1 / 2-\left|z_{n}\right|<1 / 2-1 / 2(n+$ 2) holds for at least $M$ positive integers $n$ of the set $\{1,2, \ldots, 2 M\}$.

ACKNOWLEDGEMENTS. I thank the referee of this paper for careful reading and useful corrections. This research was supported in part by the Lithuanian State Studies and Science Foundation and by INTAS grant No. 03-51-5070.

\section{REFERENCES}

1. Adamczewski, B., Bugeaud, Y., On the complexity of algebraic numbers, Ann. of Math. (to appear).

2. Adamczewski, B., Bugeaud, Y., Luca, F., Sur la complexité des nombres algébriques, C.R. Acad. Sci. Paris Sér. I 339 (2004), 11-14.

3. Akhunzhanov, R. K., Moshchevitin, N. G., On the chromatic number of a distance graph associated with a lacunary sequence, Dokl. Ross. Akad. Nauk 397 (2004), 295-296 (in Russian).

4. Akhunzhanov, R. K., Moshchevitin, N. G., Density modulo 1 of sublacunary sequences, Math. Notes 77 (2005), 741-750.

5. Akiyama, S., Mahler's Z-number and $3 / 2$ number system, preprint

6. Akiyama, S., Frougny, C., Sakarovitch, J., On number representation in a rational base (submitted).

7. Bugeaud, Y., Linear mod one transformations and the distribution of fractional parts $\left\{\xi(p / q)^{n}\right\}$, Acta Arith. 114 (2004), 301-311.

8. Bugeaud, Y., Dubickas, A., Fractional parts of powers and Sturmian words, C.R. Acad. Sci. Paris Ser. I 341 (2005), 69-74.

9. de Mathan, B., Numbers contravening a condition in density modulo 1, Acta Math. Acad. Sci. Hung. 36 (1980), 237-241. 
10. Dubickas, A., Arithmetical properties of powers of algebraic numbers, Bull. London Math. Soc. 38 (2006), 70-80.

11. Dubickas, A., On the distance from a rational power to the nearest integer, J. Number Theory 117 (2006), 222-239.

12. Dubickas, A., On the powers of $3 / 2$ and other rational numbers, Math. Nachr. (to appear).

13. Dubickas, A., Novikas, A., Integer parts of powers of rational numbers, Math. Z. 251 (2005), 635-648.

14. Erdős, P., Problems and results on Diophantine approximations. II, Repartition modulo 1, Actes Colloq. Marseille-Luminy 1974, Lecture Notes in Math. 475 (1975), 89-99.

15. Flatto, L., Lagarias, J. C., Pollington, A. D., On the range of fractional parts $\left\{\xi(p / q)^{n}\right\}$, Acta Arith. 70 (1995), 125-147.

16. Katznelson, Y., Chromatic numbers of Cayley graphs on $\mathrm{Z}$ and recurrence, Combinatorica 21 (2001), 211-219.

17. Koksma, J. F., Ein mengen-theoretischer Satz über Gleichverteilung modulo eins, Compositio Math. 2 (1935), 250-258.

18. Lothaire, M., Algebraic Combinatorics on Words, Encyclopedia Math. Appl. 90 (2002).

19. Mahler, K., An unsolved problem on the powers of 3/2, J. Austral. Math. Soc. 8 (1968), 313-321.

20. Pollington, A. D., On the density of the sequence $\left\{n_{k} \xi\right\}$, Illinois J. Math. 23 (1979), 511-515.

21. Pollington, A. D., Progressions arithmétiques généralisées et le problème des $(3 / 2)^{n}$, C.R. Acad. Sci. Paris Sér. I 292 (1981), 383-384.

22. Ruzsa, I. Z., Tuza, Zs., Voigt, M., Distance graphs with finite chromatic number, J. Combin. Theory Ser. B 85 (2002), 181-187.

23. Tijdeman, R., Note on Mahler's $\frac{3}{2}$-problem, K. Norske Vidensk. Selsk. Skr. 16 (1972), 1-4.

24. Vijayaraghavan, T., On the fractional parts of the powers of a number. I, J. London Math. Soc. 15 (1940), 159-160.

25. Weyl, H., Über die Gleichverteilung von Zahlen modulo Eins, Math. Ann. 77 (1916), 313-352.

DEPARTMENT OF MATHEMATICS AND INFORMATICS

VILNIUS UNIVERSITY

NAUGARDUKO 24, VILNIUS LT-03225

LITHUANIA

E-mail: arturas.dubickas@maf.vu.lt 